\title{
Pilot Study of Markers for High-grade Anal Dysplasia in a Southern Cohort From the Women's Interagency Human Immunodeficiency Virus Study
}

\author{
Cecile D. Lahiri, ${ }^{1,2}$ Minh Ly Nguyen, ${ }^{1,2}$ C. Christina Mehta, ${ }^{2,3}$ Marina Mosunjac, ${ }^{4}$ Talaat Tadros, ${ }^{4}$ Elizabeth R. Unger, ${ }^{5}$ Mangalathu S. Rajeevan, \\ Jendai Richards, ${ }^{5}$ Ighovwerha Ofotokun, ${ }^{1,2}$ and Lisa Flowers ${ }^{2,6}$ \\ ${ }^{1}$ Division of Infectious Diseases, Department of Medicine, Emory University School of Medicine, ${ }^{2}$ Atlanta Women's Interagency HIV Study, ${ }^{3}$ Department of Biostatistics and Bioinformatics, Rollins \\ School of Public Health, Emory University, ${ }^{4}$ Department of Pathology, Emory University School of Medicine, ${ }^{5}$ Division of High-Consequence Pathogens and Pathology, Centers for Disease Control \\ and Prevention, and ${ }^{6}$ Department of Gynecology and Obstetrics, Emory University School of Medicine, Georgia
}

\section{(See the Editorial Commentary by Susan Bennett on pages 1129-30.)}

Background. Anal cancer rates have increased, particularly in human immunodeficiency virus (HIV)-infected (HIV $\left.{ }^{+}\right)$women. We assessed factors associated with anal precancer in $\mathrm{HIV}^{+}$and at-risk HIV-negative women from the Atlanta Women's Interagency HIV Study cohort.

Methods. All participants underwent high-resolution anoscopy and anal cytology and had anal and cervical samples collected. Specimens were tested for 37 human papillomavirus (HPV) types and for FAM19A4 and microRNA124-2 promoter methylation. Binary logistic regression and multivariate analysis were conducted with histologic anal high-grade squamous intraepithelial lesion (A-HSIL) as the dependent variable.

Results. Seventy-five women were enrolled: 52 (69\%) were $\mathrm{HIV}^{+}$with three-fourths having undetectable viral load; 64 (86\%) were black; mean age was $49 \pm 8$ years. Forty-nine (65\%) anal cytology samples were abnormal, and 38 (51\%) of anal samples were positive for at least 1 of 13 high-risk HPV (hrHPV) types. Thirteen (18\%) anal biopsies identified A-HSIL. Hypermethylation of FAM19A4 and/or microRNA124-2 was found in 69 (95\%) anal samples and 19 (26\%) cervical samples. In multivariate analyses, the odds of having A-HSIL were $>6$ times higher in women with anal hrHPV (adjusted odds ratio [aOR], 6.08 [95\% confidence interval $\{\mathrm{CI}\}, 1.27-29.18], P=.02$ ) and with positive cervical methylation (aOR, 6.49 [95\% CI, 1.66-25.35], $P=.007)$, but not significantly higher in women with positive anal methylation.

Conclusions. Anal hrHPV and promoter hypermethylation in the cervix show promise as biomarkers for anal cancer screening in $\mathrm{HIV}^{+}$and at-risk HIV-negative women. Greater understanding of gene silencing by promoter hypermethylation in anal carcinogenesis is needed.

Keywords. anal cancer; high-grade squamous intraepithelial lesion (HSIL); HIV; women; methylation.

Anal cancer incidence has more than doubled during 1975-2015, from 0.8 to 1.9 cases per 100000 population [1]. Human papillomavirus (HPV) has been detected in $80 \%-90 \%$ of anal cancers [2]. Oncogenic HPV types, particularly HPV-16, are associated with anal cancer and precancer (anal high-grade squamous intraepithelial lesion [A-HSIL]) [3-6]. Human immunodeficiency virus (HIV)-infected $\left(\mathrm{HIV}^{+}\right)$women have increased risk for persistent HPV infections [7-11] and an almost 8-fold increased risk of anal precancer compared with women in the general population

Received 16 November 2018; editorial decision 9 February 2019; accepted 3 May 2019; published online May 6, 2019.

Presented in part: Meeting of the American Society for Colposcopy and Cervical Pathology, Las Vegas, Nevada, 19 April 2018.

Correspondence: L. Flowers, Emory University School of Medicine, 69 Jesse Hill Jr Drive, Atlanta, GA 30303 (Iflowe2@emory.edu).

\section{Clinical Infectious Diseases ${ }^{\circledR} \quad$ 2020;70(6):1121-8}

(C) The Author(s) 2019. Published by Oxford University Press for the Infectious Diseases Society of America. All rights reserved. For permissions, e-mail: journals.permissions@oup.com. DOI: $10.1093 /$ cid/ciz336
[12]. Mortality from anal cancer in the $\mathrm{HIV}^{+}$population remains significant, with a 64\% 5-year survival rate [13].

While no data currently show that identification and treatment of A-HSIL reduces incidence of anal cancer, the American Society for Colposcopy and Cervical Pathology recommends screening $\mathrm{HIV}^{+}$women or those with lower genital tract neoplasia using anal cytology (AC) with triage to high-resolution anoscopy (HRA)-guided biopsy and A-HSIL treatment [14]. Feasibility of performing HRA on all women with abnormal $\mathrm{AC}$ is limited due to lack of providers trained in the procedure. Alternative noninvasive markers are needed to identify women at highest risk of A-HSIL.

A meta-analysis showed that HPV tests applied to anal samples have a pooled sensitivity and specificity of $91.3 \%$ (95\% confidence interval [CI], 78.9\%-96.7\%) and 33.1\% (95\% CI, 22.2\%-46.3\%), respectively, for detecting A-HSIL or greater disease, although study populations were predominantly male [15]. DNA methylation, epigenetic modification resulting in tumor suppressor 
and DNA repair inactivation, plays an important role in cervical carcinogenesis [16]. Gene methylation has the potential to be applied as a risk biomarker in women with positive clinical HPV tests to identify those requiring colposcopy [17-21]. FAM19A4 gene promoter methylation levels in cervical scrapes of women with HPV detected cervical intraepithelial neoplasia (CIN) 3 (ie, HSIL in current terminology) with comparable sensitivity and higher specificity than cytology [21], and a recent study showed an increasing level of FAM19A4 methylation with increasing severity of cervical lesions [22]. Similarly, gene promoter methylation of micro-RNA124-2 (miR124-2) strongly predicted CIN 3 [19]. Aberrant DNA methylation patterns have been identified in anal carcinomas compared to normal tissue $[23,24]$ and in high-risk (tumor $>5 \mathrm{~cm}$ and/or nodal involvement) vs low-risk anal cancers [25]. However, methylation markers have not been explored in anal cancer screening strategies.

HPV exposure at 1 genital site increases risk for exposure at others, so women with cervical HPV may be more likely to have anal HPV, and HPV oncogenic changes may affect multiple genital sites (the "field effect") [26]. In high-risk women, cervical findings may be sufficient to guide HRA referral. We assessed cytology, HPV genotyping, and DNA methylation of FAM19A4 and miR124-2 promoter regions, as predictors of A-HSIL/cancer in a population of $\mathrm{HIV}^{+}$and at-risk HIVnegative (ARHIVN) women.

\section{MATERIALS AND METHODS}

\section{Study Population}

A cross-sectional pilot study was conducted within the Atlanta site of the Women's Interagency HIV Study (WIHS), a cohort created in 2013 consisting of two-thirds $\mathrm{HIV}^{+}$and one-third ARHIVN women. Women were classified as ARHIVN if they or their sexual partner reported $\geq 1$ criteria within 5 years of enrollment: (1) intravenous or other illicit drug use; (2) sexually transmitted infection; (3) unprotected sex with $\geq 3$ partners; (4) sex with $\geq 6$ partners; (5) transactional sex; or (6) sex with known $\mathrm{HIV}^{+}$partner. WIHS data collection and methods have been previously described [27]; in brief, participants complete questionnaires, blood draws, and physical/gynecological examinations during semiannual visits. All women within WIHS undergo colposcopy if they have (1) visible lesion(s) concerning for condyloma; (2) atypical squamous cells of undetermined significance (ASCUS) on cervical cytology without prior colposcopy; or (3) more significant cervical cytologic abnormalities, that is, low-grade squamous intraepithelial lesion (LSIL) or more severe, or atypical glandular cells. For this substudy, eligibility criteria included (1) no history of A-HSIL or anal cancer; (2) no active anal/rectal disease necessitating surgery; and (3) willing/ able to undergo cervical/anal sampling and HRA. Participants were enrolled after informed consent obtained prior to the study visit. The Emory Institutional Review Board and the Grady Research Oversight Committee approved the study.

\section{Data Collection}

Data collected from core WIHS visits were abstracted and included sociodemographics, smoking status, alcohol consumption, sexual history, cervical cytology and histology, CD4 cell count, and HIV RNA load within 12 months of the study visit. Additional data collected at the study visit included cervical and anal HPV genotyping, FAM19A4 and miR124-2 gene promoter methylation, and anal cytology and histology.

\section{Study Procedures and Specimen Collection}

Investigators collected cervical and anal swabs prior to HRA (Figure 1). A cytobroom was rotated 3 times in the cervical os and agitated in a ThinPrep vial 3-5 times. Two saline-moistened Dacron swabs were sequentially inserted 3-5 cm into the anal canal and rotated for 30 seconds. The first anal swab was agitated for 15 seconds in SurePath liquid media for AC, and the second swab was placed in ThinPrep media for HPV genotyping and methylation analysis.

HRA was performed on all participants by a trained anoscopist. Abnormal anal areas were identified using 3\% acetic acid and Lugol solution, biopsied, and placed in buffered formalin solution. AC and histology specimens were transported to the Grady Memorial Hospital Pathology Laboratory at room temperature within 24 hours of collection. Anal and cervical ThinPrep specimens were maintained at $4^{\circ} \mathrm{C}$ and transported to the Centers for Disease Control and Prevention within 72 hours of collection.

\section{Anal Cytology and Histology}

All AC and histology specimens were interpreted by 2 pathologists with expertise in anal histopathology. AC was classified based on 2001 and 2014 Bethesda terminology for cervical cytology $[28,29]$ : NILM (negative for intraepithelial lesion and malignancy), ASCUS, LSIL, ASC-H (atypical squamous cells, cannot exclude HSIL), and HSIL. Anal histology results were reported using Lower Anogenital Squamous Terminology (LAST) classification [30]: benign/ negative, LSIL (condyloma or anal intraepithelial neoplasia [AIN] grade 1), HSIL (AIN grade 2 or 3 ), or invasive squamous cell carcinoma. P16 staining was performed per LAST recommendations [30].

\section{Extraction and Molecular Testing}

Cells from a $12-\mathrm{mL}$ aliquot of each sample were pelleted by centrifugation. Collection media was decanted and pellets were stored at $-80^{\circ} \mathrm{C}$ until extraction using the Roche MagNA Pure LC instrument with the MagNA Pure LC DNA Isolation Kit III and external lysis step (Roche Applied Science, Mannheim, Germany). Each thawed pellet was suspended in $130 \mu \mathrm{L}$ lysis buffer and $20 \mu \mathrm{L}$ proteinase $\mathrm{K}$ from MagNA Pure LC reagents. Samples were incubated at $65^{\circ} \mathrm{C}$ for 1 hour and then added to the instrument, yielding $100 \mu \mathrm{L}$ final extract volume. Extracts were maintained at $4^{\circ} \mathrm{C}$ for up to a week and stored at $-80^{\circ} \mathrm{C}$. 


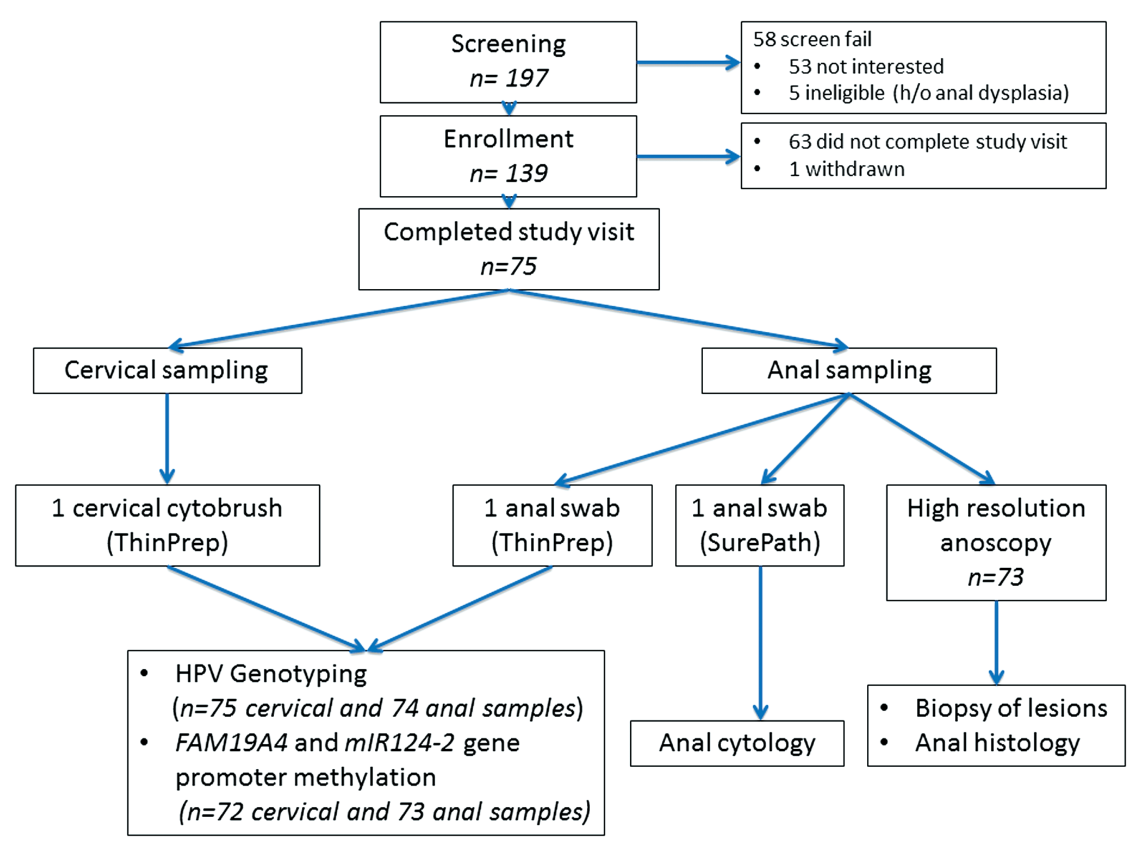

Figure 1. Study schema ( $N=75$ unless otherwise noted). Abbreviations: $h / 0$, history of; HPV, human papillomavirus.

HPV detection and typing were performed with Research Use Only Linear Array HPV Genotyping Test (LA; Roche Molecular Diagnostics, Indianapolis, Indiana) as previously described [31]. The manufacturer's protocol was modified to use $5 \mu \mathrm{L}$ per $100 \mu \mathrm{L}$ reaction, and type-specific hybridization was automated. The LA test relies on L1 consensus polymerase chain reaction (PCR) and includes primers for $\beta$-globin to control for specimen adequacy. Type-specific hybridization allows qualitative detection of 36 types, HPV-52 with potential cross-reactivity, and positive control ( $\beta$-globin). Ambiguous results for HPV-52 are resolved with a type-specific quantitative PCR [32]. Samples testing negative for HPV and $\beta$-globin were considered inadequate. Thirteen of the $14 \mathrm{HPV}$ types included in clinical assays were considered high risk (hrHPV): 16, 18, 31, $33,35,39,45,51,52,56,58,59,68$, omitting HPV-66, which is not in International Agency for Research on Cancer HPV carcinogenicity group 1 or $2 \mathrm{~A}[33,34]$. Additional HPV types detected by LA are $6,11,26,40,42,53,54,55,61,62,64,66,67$, $69,70,71,72,73,81,82,83,84,89$, and IS39.

DNA (1.6-250 ng), quantified by Qubit Fluorometer (Life Technologies, Carlsbad, California), was treated with sodium bisulfite via the EZ DNA Methylation Kit (Zymo Research, Irvine, California) and collected in $12.5 \mu \mathrm{L}$ elution buffer. The PrecursorM+ kit from Self-Screen (www.self-screen.nl), a realtime multiplex methylation-specific PCR assay, was used for detection of FAM19A4 and miR124-2 promoter methylation level. PreCursor M+ uses primers and probes specific for FAM19A4 and miR124-2, and for reference gene $\beta$-actin (ACTB) as sample quality control. Positive controls for all 3 genes serve as the calibrator for expressing differential methylation. PCR reactions were performed on the Rotorgene-Q5-plex thermal cycler (Qiagen) following the Precursor M+ kit protocol using $2.5 \mu \mathrm{L}$ of bisulfite-treated DNA. Promoter methylation results were reported as the delta-delta $(\Delta \Delta)$ cycle threshold (Ct) for each marker gene [35]. Methylation levels of target genes were normalized to $A C T B$ and expressed relative to the calibrator. Clinical samples were considered methylation positive when the $\triangle \triangle \mathrm{Ct}$ for FAM19A4 was $<10.36$ and/or $<6.50$ for miR124-2, per the manufacturer's instructions. Repeat assays for 28 (19\%) samples showed significant correlation of Ct using Pearson coefficient analyses: $F A M 19 A 4=0.92$ and $m i R 124-2=0.96$.

\section{Statistical Analyses}

Data were summarized using descriptive statistics with counts and percentages for categorical variables, and mean \pm standard deviation or median with first and third quartiles for continuous variables. Statistical comparisons between $\mathrm{HIV}^{+}$vs ARHIVN or between women with histologic A-HSIL vs other used $\chi^{2}$ or Fisher exact tests for categorical variables and 2-sided 2-sample $t$ tests or Wilcoxon rank-sum tests for continuous variables. Variables significant at $P<.2$ in bivariate analyses for histologic A-HSIL were included in multivariate analyses. Models using a penalized $\log$-likelihood were created using backward selection $(P<.1)$ of variables where the event of interest was histologic A-HSIL.

\section{RESULTS}

\section{Demographic and Clinical Characteristics}

One hundred ninety-seven (197) women within Atlanta WIHS were screened, 139 were enrolled, and 75 completed the study and were included in the analyses (Figure 1). Fifty-two (69\%) 
were $\mathrm{HIV}^{+}$and 23 (31\%) were ARHIVN. Mean age of women was $48.7 \pm 8.5$ years. Women were predominantly black (86.5\%), had high school education or less (70.3\%), and had annual incomes $\leq \$ 12000$ (57.5\%). Among $\mathrm{HIV}^{+}$women, 50 (96\%) had CD4 count $>200$ cells $/ \mu \mathrm{L}$ and $40(77 \%)$ had HIV RNA $<20$ copies $/ \mathrm{mL}$ within the last 6 months. $\mathrm{HIV}^{+}$women were more likely to have health insurance (including Ryan White funding) than ARHIVN (96.2\% vs $59.1 \%, P=.0002)$ but were similar in other sociodemographic characteristics (Table 1).

\section{Anal Cytology and Histology}

Forty-nine women $(65.3 \%)$ had abnormal anal cytology: 34 (45.3\%) ASCUS, 11 (14.7\%) LSIL, and 4 (5.3\%) ASC-H, and this distribution was similar across HIV status. Thirty-seven (50.7\%) women had normal HRA findings: 20 (27.4\%) had a negative HRA exam without biopsies and 17 (23.3\%) had normal biopsy results. Of the 36 (49.3\%) women with abnormal anal histology, 23 (31.5\%) had LSIL/condyloma and 13 (17.8\%) had HSIL (Table 2). Anal histology findings did not differ between $\mathrm{HIV}^{+}$and ARHIVN women.

\section{Cervical Cytology and Histology From WIHS Core Visit}

Most women had normal cervical cytology within 12 months of the study visit, and this was similar by HIV status: $80.8 \%$ vs 95.7\% of $\mathrm{HIV}^{+}$and ARHIVN women, respectively. Only 23 women $(30.7 \%)$ underwent colposcopy within 12 months of the study visit: 13 (56.5\%) had normal biopsy results, 9 (39.1\%) had LSIL, and 1 (4.3\%) had HSIL. HIV ${ }^{+}$women were more likely to undergo colposcopy and have abnormal biopsy results than ARHIVN women: $19(82.6 \%)$ vs $4(17.4 \%)$ and 10 of $10(100 \%)$ vs 0 , respectively $(P=.05$; Table 2$)$.

\section{Cervical and Anal HPV}

Forty-nine (65.3\%) women had detectable cervical HPV; 20 (26.7\%) had hrHPV with 3 (4.0\%) having HPV-16/18, and this was similar by HIV status. Overall, 57 (77.0\%) of women had detectable anal HPV; 36 (48.7\%) had hrHPV with 13 (17.6\%) having HPV-16/18 detected. HIV ${ }^{+}$women were more likely to have anal HPV-16/18 detected than ARHIVN women (23.5 vs $4.4 \%, P=.05$; Table 2).

\section{Cervical and Anal Methylation of FAM19A4 and miR124-2}

One hundred forty-eight of 150 samples (98.7\%) had sufficient DNA and were evaluable for cervical and anal methylation. Of those, 98\% (145/148) met quality criteria, defined as Ct value $\leq 30.0$ for control gene $A C T B$, in concordance with published literature [20]. At least 1 gene promoter region (FAM19A4 or miR124-2) was considered methylation positive in cervical samples from 19 (26.4\%) women. This proportion did not differ by HIV status. In contrast, nearly all (69 [94.5\%]) anal samples were methylation positive for at least 1 target (Table 2).

\section{Predictors of Histologic Anal HSIL}

Compared to women without histologic A-HSIL $(n=60)$, those with A-HSIL $(\mathrm{n}=13)$ were more likely to drink $>7$

Table 1. Demographic and Clinical Characteristics

\begin{tabular}{|c|c|c|c|c|}
\hline Characteristic & Overall $(\mathrm{N}=75)^{\mathrm{a}}$ & HIV-infected $(n=52)$ & HIV-negative $(n=23)$ & $P$ Value $^{\mathrm{b}}$ \\
\hline Age, $y$, mean $\pm S D$ & $48.7 \pm 8.5$ & $49.1 \pm 8.1$ & $47.7 \pm 9.6$ & .06 \\
\hline Race/ethnicity ( $n=74$ ) & & & & .47 \\
\hline Black & $64(86.5)$ & $46(88.5)$ & $18(81.8)$ & \\
\hline White & $4(5.4)$ & $3(5.8)$ & $1(4.6)$ & \\
\hline Hispanic & $3(4.0)$ & $2(3.9)$ & $1(4.6)$ & \\
\hline Other & $3(4.0)$ & $1(1.9)$ & $2(9.1)$ & \\
\hline High school education or less $(n=74)$ & $52(70.3)$ & $37(71.2)$ & $15(68.2)$ & .79 \\
\hline Income $\leq \$ 12$ 000/y $(n=73)$ & $42(57.5)$ & $31(60.8)$ & $11(50.0)$ & .39 \\
\hline Unemployed $(n=74)$ & $54(73.0)$ & $41(78.9)$ & $13(59.1)$ & .08 \\
\hline Has health insurance $(n=74)$ & $63(85.1)$ & $50(96.2)$ & $13(59.1)$ & .0002 \\
\hline Alcohol consumption $(n=74)$ & & & & .58 \\
\hline Abstainer & $30(40.5)$ & $22(42.3)$ & $8(36.4)$ & \\
\hline 0-7 drinks/week & $35(47.3)$ & $25(48.1)$ & $10(45.5)$ & \\
\hline >7 drinks/week & $9(12.2)$ & $5(9.6)$ & $4(18.2)$ & \\
\hline Current smoker $(n=74)$ & $43(58.1)$ & $30(57.7)$ & $13(59.1)$ & .91 \\
\hline History of anal sex (ever) & $16(21.3)$ & $11(21.2)$ & $5(21.7)$ & 1.00 \\
\hline Male partners in last 6 mo, median, IOR & $1(0-1)$ & $1(1-2)$ & $1(0-1)$ & .10 \\
\hline CD4 count $>200$ cells $/ \mu \mathrm{L}$ & NA & $50(96.2)$ & NA & NA \\
\hline HIV RNA <20 copies/mL & NA & $40(76.9)$ & NA & NA \\
\hline
\end{tabular}

Data are presented as No. (\%) unless otherwise indicated. Values in bold indicate statistical significance at $P<.05$.

Abbreviations: HIV, human immunodeficiency virus; IQR, interquartile range; NA, not applicable; SD, standard deviation.

${ }^{a}$ Data available for 75 women unless otherwise noted.

${ }^{b}$ Statistical comparison conducted using $\chi^{2}$ or Fisher exact tests for categorical variables and 2 -sided 2-sample $t$ tests or Wilcoxon rank-sum tests for continuous variables. 
Table 2. Anal and Cervical Characteristics by Human Immunodeficiency Virus Status ${ }^{\mathrm{a}}$

\begin{tabular}{|c|c|c|c|c|}
\hline Characteristic & Overall $\left(\mathrm{N}=75^{\mathrm{a}}\right)$ & HIV-infected $(n=52)$ & HIV-negative $(n=23)$ & $P$ Value $^{\mathrm{b}}$ \\
\hline Anal cytology & & & & .61 \\
\hline NILM & $26(34.7)$ & $19(36.5)$ & $7(30.4)$ & \\
\hline ASCUS & 34 (45.3) & $21(40.4)$ & $13(56.5)$ & \\
\hline LSIL & $11(14.7)$ & $9(17.3)$ & $2(8.7)$ & \\
\hline ASC-H & $4(5.3)$ & $3(5.8)$ & $1(4.4)$ & \\
\hline Anal histology $(n=73)$ & & & & .85 \\
\hline No biopsies & $20(27.4)$ & $15(30.0)$ & $5(21.7)$ & \\
\hline Benign & 17 (23.3) & $11(22.0)$ & $6(26.1)$ & \\
\hline LSIL/condyloma & $23(31.5)$ & $16(32.0)$ & $7(30.4)$ & \\
\hline HSIL & $13(17.8)$ & $8(16.0)$ & $5(21.7)$ & \\
\hline \multicolumn{5}{|l|}{ Anal HPV $(n=74)$} \\
\hline Any & $57(77.0)$ & $42(82.4)$ & $15(65.2)$ & .10 \\
\hline hrHPV & $36(48.7)$ & $28(54.9)$ & 8 (34.8) & .11 \\
\hline HPV-16/18 & $13(17.6)$ & $12(23.5)$ & $1(4.4)$ & .05 \\
\hline \multicolumn{5}{|l|}{ Anal methylation positive $(n=73)$} \\
\hline Any (FAM19A4 or miR124-2) & 69 (94.5) & $49(98.0)$ & $20(87.0)$ & .09 \\
\hline FAM19A4 only & 69 (94.5) & $36(72.0)$ & $16(69.6)$ & .09 \\
\hline miR124-2 only & $52(71.2)$ & $49(98.0)$ & $20(87.0)$ & 1.00 \\
\hline Cervical cytology & & & & .47 \\
\hline NILM & $64(85.3)$ & $42(80.8)$ & $22(95.7)$ & \\
\hline ASCUS & $7(9.3)$ & $6(11.5)$ & $1(4.4)$ & \\
\hline LSIL & $2(2.7)$ & $2(3.9)$ & $0(0)$ & \\
\hline ASC-H & $2(2.7)$ & 2 (3.9) & $0(0)$ & \\
\hline HSIL & $0(0)$ & $0(0)$ & $0(0)$ & \\
\hline Cervical histology & & & & .05 \\
\hline No colposcopy performed & 49 (65.3) & $31(59.6)$ & $18(78.3)$ & \\
\hline Normal & $13(17.3)$ & $9(17.3)$ & $4(17.4)$ & \\
\hline LSIL & $9(12.5)$ & $9(17.3)$ & 0 & \\
\hline HSIL & $1(1.4)$ & $1(2.0)$ & 0 & \\
\hline Missed $^{d}$ & $3(4.0)$ & $2(3.9)$ & $1(4.4)$ & \\
\hline \multicolumn{5}{|l|}{ Cervical HPV } \\
\hline Any & $49(65.3)$ & $36(69.2)$ & $13(56.5)$ & .30 \\
\hline hrHPV & $20(26.7)$ & $16(30.8)$ & $4(17.4)$ & .23 \\
\hline HPV-16/18 & $3(4.0)$ & $3(5.8)$ & $0(0)$ & .55 \\
\hline \multicolumn{5}{|c|}{ Cervical methylation positive $(n=72)$} \\
\hline Any (FAM19A4 or miR124-2) & $19(26.4)$ & $13(25.5)$ & $6(28.6)$ & .78 \\
\hline FAM19A4 only & $19(26.4)$ & $13(25.5)$ & $6(28.6)$ & .78 \\
\hline miR124-2 only & $5(6.9)$ & $4(7.8)$ & $1(4.8)$ & 1.00 \\
\hline
\end{tabular}

Data are presented as No. (\%) unless otherwise indicated. Values in bold indicate statistical significance at $P<.05$.

Abbreviations: ASC-H, atypical squamous cells, cannot exclude high-grade squamous intraepithelial lesion; ASCUS, atypical squamous cells of undetermined significance; HIV, human immunodeficiency virus; HPV, human papillomavirus; hrHPV, high-risk human papillomavirus; HSIL, high-grade squamous intraepithelial lesion; LSIL, low-grade squamous intraepithelial lesion; NILM, negative for intraepithelial lesion or malignancy.

${ }^{a}$ Data available for 75 women unless otherwise noted.

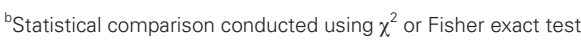

${ }^{\circ}$ Column does not equal $100 \%$ due to hrHPV and HPV-16/18 being subcategories of any HPV.

${ }^{d}$ Colposcopy indicated due to abnormal cervical cytology but missed appointment.

alcoholic beverages per week (30.8\% vs $8.5 \%, P=.01)$ and to have detectable anal hrHPV ( $84.6 \%$ vs $42.4 \%, P=.01)$, in particular HPV-16/18 (38.5\% vs $13.6 \%, P=.05)$, Table 3. Twelve women with A-HSIL (92.3\%) had $\geq 1$ anal HPV type detected, and $11(84.6 \%)$ had $\geq 1$ anal hrHPV type. Four women with A-HSIL (30.8\%) had HPV-16, 8 (61.5\%) had HPV types 45, 51, 52 , or 68 , and $2(15.4 \%)$ had HPV-18. Eleven women (84.6\%) with A-HSIL had cervical HPV detected, and 6 (46.2\%) had $\geq 1$ hrHPV type. Six women with A-HSIL (46.2\%) had $\geq 1$ concordant HPV type in the anus and cervix, and $3(50 \%)$ had concordant hrHPV types. Eleven women (84.6\%) with A-HSIL had $\geq 1$ type included in the 9-valent HPV vaccine $(6,11,16$, $18,31,33,45,52,58)$.

Women with A-HSIL were more likely to have abnormal cervical cytology $(38.5 \%$ vs $10.0 \%, P=.007)$, abnormal cervical histology ( $30.8 \%$ vs $10.0 \%, P=.05)$, and positive cervical methylation $(61.5 \%$ vs $19.3 \%, P=.004)$ (Table 3). In contrast, positive anal methylation for FAM19A4 or miR124-2 did not 
Table 3. Univariable Results: Histologic Anal High-grade Squamous Intraepithelial Lesion Versus Other ${ }^{a}$

\begin{tabular}{|c|c|c|c|}
\hline Characteristic & Anal HSIL ( $n=13)$ & Other $(n=60)$ & $P$ Value ${ }^{b}$ \\
\hline HIV infected & $8(61.5)$ & $42(70.0)$ & .53 \\
\hline Age, $y$, mean $\pm S D$ & $48.5 \pm 9.2$ & $48.6 \pm 8.4$ & 1.0 \\
\hline Race/ethnicity ${ }^{c}$ & & & .41 \\
\hline Black & $11(84.6)$ & $52(88.1)$ & \\
\hline White & $1(7.7)$ & $3(5.1)$ & \\
\hline Hispanic & $0(0)$ & $3(5.1)$ & \\
\hline Other & $1(7.7)$ & $1(1.7)$ & \\
\hline Alcohol consumption ${ }^{\mathrm{d}}$ & & & .01 \\
\hline Abstainer & $1(7.7)$ & $28(47.5)$ & \\
\hline 0-7 drinks/week & $8(61.5)$ & $26(44.1)$ & \\
\hline$>7$ drinks/week & $4(30.8)$ & $5(8.5)$ & \\
\hline Current smoker ${ }^{\mathrm{c}}$ & $10(76.9)$ & $31(52.4)$ & .13 \\
\hline History of anal sex (ever) & $3(23.1)$ & $12(20.0)$ & .72 \\
\hline Male partners in last 6 mo, median, IQR & $1(1-2)$ & $1(0-1)$ & .10 \\
\hline Anal cytology & & & .10 \\
\hline Normal & $2(15.4)$ & $23(38.3)$ & \\
\hline ASCUS/LSIL & $9(69.2)$ & $35(58.3)$ & \\
\hline ASC-H & $2(15.4)$ & 2 (3.3) & \\
\hline \multicolumn{4}{|l|}{ Anal HPV } \\
\hline Any HPV & $12(92.3)$ & $44(74.6)$ & .27 \\
\hline hrHPV & $11(84.6)$ & $25(42.4)$ & .01 \\
\hline HPV-16/18 & 5 (38.5) & $8(13.6)$ & .05 \\
\hline Anal methylation positive ${ }^{\mathrm{C}}$ (FAM19A4 or miR124-2) & $11(84.6)$ & 57 (96.6) & .15 \\
\hline Cervical cytology & & & .007 \\
\hline Normal & $8(61.5)$ & $54(90.0)$ & \\
\hline ASCUS & $3(23.1)$ & $4(6.7)$ & \\
\hline LSIL & $2(15.4)$ & $0(0)$ & \\
\hline ASC-H/HSIL & $0(0)$ & $2(3.3)$ & \\
\hline Cervical colposcopy & & & .05 \\
\hline No colposcopy & $5(38.5)$ & $43(71.7)$ & \\
\hline Normal & $4(30.8)$ & $8(13.3)$ & \\
\hline LSIL/condyloma & $4(30.8)$ & $5(8.3)$ & \\
\hline HSIL & $0(0)$ & $1(1.7)$ & \\
\hline Missed $^{d}$ & $0(0)$ & $3(5.0)$ & \\
\hline \multicolumn{4}{|l|}{ Cervical HPV } \\
\hline Any HPV & $11(84.6)$ & $36(60.0)$ & .12 \\
\hline hrHPV & $6(46.2)$ & $14(23.3)$ & .17 \\
\hline HPV-16/18 & $2(15.4)$ & $1(1.7)$ & .08 \\
\hline Cervical methylation positive ${ }^{e}$ (FAM19A4 or miR124-2) & $8(61.5)$ & $11(19.3)$ & .004 \\
\hline
\end{tabular}

Data are presented as No. (\%) unless otherwise indicated. Values in bold indicate statistical significance at $P<.05$.

Abbreviations: ASC-H, atypical squamous cells, cannot exclude high-grade squamous intraepithelial lesion; ASCUS, atypical squamous cells of undetermined significance; HIV, human immunodeficiency virus; HPV, human papillomavirus; hrHPV, high-risk human papillomavirus; HSIL, high-grade squamous intraepithelial lesion; IQR, interquartile range; LSIL, low-grade squamous intraepithelial lesion; SD, standard deviation.

a "Other" indicates anoscopy result other than HSIL, including no biopsies, benign, condyloma, or LSIL.

${ }^{b}$ Statistical comparison conducted using $\chi^{2}$ or Fisher exact tests for categorical variables and 2 -sided 2-sample $t$ tests or Wilcoxon rank-sum tests for continuous variables.

"Data available for 59 women in "Other" group.

${ }^{\mathrm{d} C}$ Colposcopy indicated due to abnormal cervical cytology but missed appointment.

eData available for 57 women in "Other" group.

differ significantly between women with and without A-HSIL: $84.6 \%$ vs $96.6 \%$, respectively. In multivariate analyses, the odds of having A-HSIL were $>6$ times higher in women with anal hrHPV (adjusted odds ratio [aOR], 6.08 [95\% CI, 1.27-29.18], $P=.02$ ) and with positive cervical methylation (aOR, $6.49[95 \%$ CI, 1.66-25.35], $P=.007$ ) (Table 4).

\section{DISCUSSION}

This pilot study evaluated predictive markers for anal precancer (A-HSIL) in a southern US cohort of $\mathrm{HIV}^{+}$and ARHIVN women. To our knowledge, this is the first study to assess association of both cervical and anal DNA methylation of FAM19A4 and miR124-2 gene promoters with A-HSIL. 


\begin{tabular}{lccr}
\hline Variable & Estimate & SE & OR (95\% CI) \\
\hline Anal hrHPV (ref: No) & 1.80 & 0.80 & .024 \\
$\begin{array}{l}\text { Cervical methylation positive } \\
\quad \text { (FAM19A4 or miR124-2) (ref: No) }\end{array}$ & 1.87 & 0.69 & .007 \\
\hline
\end{tabular}

Abbreviations: $\mathrm{Cl}$, confidence interval; hrHPV, high-risk human papillomavirus; OR, odds ratio; ref, reference; SE, standard error.

${ }^{a}$ Model of best fit created using a penalized log-likelihood with backward selection $(P<.1)$ of variables where the event of interest was histologic anal high-grade squamous intraepithelial lesion.

Almost two-thirds of participants had abnormal anal cytology and $18 \%$ had histologic A-HSIL, which did not differ by HIV status. Prior published data of WIHS participants from cities outside the southern United States (Chicago, San Francisco, New York) showed lower rates, with only $31 \%$ of $\mathrm{HIV}^{+}$and $8 \%$ of ARHIVN women having abnormal anal cytology and histology [5]. While our $\mathrm{HIV}^{+}$participants had similar high rates of detectable anal and cervical hrHPV ( $>80 \%$ and $>50 \%$, respectively) as previously published WIHS data, we saw much higher anal and cervical hrHPV rates in our ARHIVN population: $34.8 \%$ vs $17 \%$ and $17.4 \%$ vs $8 \%$, respectively [5]. Reasons for discrepant findings between studies may include different cohort characteristics (older age and lower socioeconomic status in our cohort) as well as the fact that the southern United States is disproportionately affected with the highest rates of HIV-associated morbidity and mortality in the nation [36].

Factors associated with A-HSIL in bivariate analyses included $>7$ alcoholic beverages weekly, abnormal cervical cytology and histology within 12 months, detectable anal hrHPV (particularly HPV-16/18), and positive cervical DNA methylation of either FAM19A4 or miR124-2 gene promoter regions. Both anal hrHPV and positive cervical methylation markers remained statistically significant predictors in multivariable logistic regression analyses with 6-fold increased odds of A-HSIL.

Frequent alcohol consumption has been associated with persistent cervical hrHPV in Korean women [37] and increased prevalence of genital hrHPV in men, likely due to impaired host defenses [38]. Previous studies show that women with history of high-grade cervical neoplasia and cervical cancer [39] have higher anal cancer risk. The association between positive cervical methylation markers for FAM19A4 or miR124-2 and A-HSIL is new and likely reflects concurrent cervical disease and/or the field effect of HPV infection and oncogenesis. Only 1 (1.4\%) woman had cervical HSIL, whereas 1 or more cervical methylation markers were positive in 19 (26.4\%) of participants. Several factors may contribute to this discrepancy. Colposcopy findings from the prior 12 months may not accurately reflect current disease. In addition, the positive threshold established by the manufacturer may need recalibration in this older US population.

Consistent with prior data, detectable anal hrHPV was significantly associated with A-HSIL [5]. While the utility of using anal hrHPV detection as an anal cancer screening strategy in men is unclear given high anal hrHPV prevalence rates (particularly in men who have sex with men), anal hrHPV testing may be a viable option for screening high-risk women. The fact that $>80 \%$ of women with A-HSIL had $\geq 1$ vaccine-preventable HPV type in the anus reaffirms the need for primary prevention, including increasing HPV vaccine availability to vulnerable populations. Most women in our study were older than the routine age established for catch-up vaccination, but vaccination may be worth considering to reduce risk in this population.

The high prevalence of positive methylation of FAM19A4 and/or miR124-2 in anal DNA resulted in a lack of correlation with A-HSIL. Anal samples may include more keratinized cells than cervical samples, and cellular differentiation is known to impact methylation. Methylation of other host genes may be more reflective of anal oncogenesis.

Our study had several limitations. First, our sample size was small and included relatively few cases of A-HSIL, limiting our ability to conduct extensive multivariable analyses. In addition, the cross-sectional design prevented us from following anal disease over time, as almost one-fourth of A-HSIL may regress spontaneously in men [40]. Data from this pilot study will support a larger longitudinal study to identify women at highest risk for developing clinically significant A-HSIL. Furthermore, to minimize participant risk, cervical cytology and histology data were retrospectively collected from WIHS core visits, which may not accurately reflect current cervical pathology. Nonetheless, by limiting analysis of cervical cytology and histology data to within 12 months of the study visit and assessing cervical DNA methylation markers, these data represent a robust assessment of cervical disease.

In conclusion, detection of anal hrHPV and/or positive promoter methylation of FAM19A4/miR124-2 genes in cervical samples appear to be promising noninvasive screening strategies for A-HSIL in both $\mathrm{HIV}^{+}$and ARHIVN women. Greater understanding of gene silencing by promoter methylation in anal carcinogenesis is needed.

\section{Notes}

Acknowledgments. The authors thank the study staff and participants of the Atlanta Women's Interagency HIV Study (WIHS) cohort for their time and dedication to this study. Data in this manuscript were collected by the WIHS.

Disclaimer. The contents of this publication are solely the responsibility of the authors and do not represent the official views of the National Institutes of Health (NIH), the Centers for Disease Control and Prevention, or the other funding agencies. 
Financial support. This work was supported by the NIH (grant numbers U-01 AI103408, KL2TR000455, UL1TR000454, P30 AI050409); the National Institute of Allergy and Infectious Diseases (NIAID; grant number K23AI124913 to C. D. L); and WIHS (principal investigators Ighovwerha Ofotokun and Gina Wingood): Atlanta WIHS (grant number U01-AI-103408). The WIHS is funded primarily by the National Institute of Allergy and Infectious Diseases, with additional co-funding from the Eunice Kennedy Shriver National Institute of Child Health and Human Development, the National Cancer Institute, the National Institute on Drug Abuse, and the National Institute of Mental Health. Targeted supplemental funding for specific projects is also provided by the National Institute of Dental and Craniofacial Research, the National Institute on Alcohol Abuse and Alcoholism, the National Institute on Deafness and other Communication Disorders, and the NIH Office of Research on Women's Health. WIHS data collection is also supported by grant number UL1-TR000454 (Atlanta CTSA).

Potential conflicts of interest. The authors: No reported conflicts of interest. All authors have submitted the ICMJE Form for Disclosure of Potential Conflicts of Interest. Conflicts that the editors consider relevant to the content of the manuscript have been disclosed.

\section{References}

1. Noone AM, Howlader N, Krapcho M, et al. SEER Cancer Statistics Review, 1975 2015. Available at: https://seer.cancer.gov/csr/1975_2015. Accessed 31 May 2018.

2. Abramowitz L, Jacquard AC, Jaroud F, et al. Human papillomavirus genotype distribution in anal cancer in France: the EDiTH V study. Int J Cancer 2011; 129:433-9

3. Conley L, Bush T, Darragh TM, et al; Study to Understand the Natural History of HIV and AIDS in the Era of Effective Therapy (SUN Study) Investigators. Factors associated with prevalent abnormal anal cytology in a large cohort of HIV-infected adults in the United States. J Infect Dis 2010; 202:1567-76.

4. Frisch M, Fenger C, van den Brule AJ, et al. Variants of squamous cell carcinoma of the anal canal and perianal skin and their relation to human papillomaviruses. Cancer Res 1999; 59:753-7.

5. Hessol NA, Holly EA, Efird JT, et al. Anal intraepithelial neoplasia in a multisite study of HIV-infected and high-risk HIV-uninfected women. AIDS 2009; 23:59-70.

6. Palefsky JM, Holly EA, Gonzales J, Berline J, Ahn DK, Greenspan JS. Detection of human papillomavirus DNA in anal intraepithelial neoplasia and anal cancer. Cancer Res 1991; 51:1014-9.

7. de Sanjosé S, Palefsky J. Cervical and anal HPV infections in HIV positive women and men. Virus Res 2002; 89:201-11.

8. Jamieson DJ, Duerr A, Burk R, et al; HIV Epidemiology Research Study (HERS) Group. Characterization of genital human papillomavirus infection in women who have or who are at risk of having HIV infection. Am J Obstet Gynecol 2002; 186:21-7.

9. Minkoff H, Feldman J, DeHovitz J, Landesman S, Burk R. A longitudinal study of human papillomavirus carriage in human immunodeficiency virus-infected and human immunodeficiency virus-uninfected women. Am J Obstet Gynecol 1998; 178:982-6.

10. Strickler HD, Burk RD, Fazzari M, et al. Natural history and possible reactivation of human papillomavirus in human immunodeficiency virus-positive women. J Natl Cancer Inst 2005; 97:577-86.

11. Sun XW, Kuhn L, Ellerbrock TV, Chiasson MA, Bush TJ, Wright TC Jr. Human papillomavirus infection in women infected with the human immunodeficiency virus. N Engl J Med 1997; 337:1343-9.

12. Frisch M, Biggar RJ, Goedert JJ. Human papillomavirus-associated cancers in patients with human immunodeficiency virus infection and acquired immunodeficiency syndrome. J Natl Cancer Inst 2000; 92:1500-10.

13. Stier EA, Sebring MC, Mendez AE, Ba FS, Trimble DD, Chiao EY. Prevalence of anal human papillomavirus infection and anal HPV-related disorders in women: a systematic review. Am J Obstet Gynecol 2015; 213:278-309.

14. Moscicki AB, Darragh TM, Berry-Lawhorn JM, et al. Screening for anal cancer in women. J Low Genit Tract Dis 2015; 19:S27-42.

15. Clarke MA, Wentzensen N. Strategies for screening and early detection of anal cancers: a narrative and systematic review and meta-analysis of cytology, HPV testing, and other biomarkers. Cancer Cytopathol 2018; 126:447-60.
16. Jones PA, Baylin SB. The epigenomics of cancer. Cell 2007; 128:683-92.

17. De Strooper LM, Hesselink AT, Berkhof J, et al. Combined CADM1/MAL methylation and cytology testing for colposcopy triage of high-risk HPV-positive women. Cancer Epidemiol Biomarkers Prev 2014; 23:1933-7.

18. De Strooper LM, van Zummeren M, Steenbergen RD, et al. CADM1, MAL and miR124-2 methylation analysis in cervical scrapes to detect cervical and endometrial cancer. J Clin Pathol 2014; 67:1067-71.

19. De Vuyst H, Franceschi S, Plummer M, et al. Methylation levels of CADM1, MAL, and MIR124-2 in cervical scrapes for triage of HIV-infected, high-risk HPVpositive women in Kenya. J Acquir Immune Defic Syndr 2015; 70:311-8.

20. Luttmer R, De Strooper LM, Dijkstra MG, et al. FAM19A4 methylation analysis in self-samples compared with cervical scrapes for detecting cervical (pre)cancer in HPV-positive women. Br J Cancer 2016; 115:579-87.

21. Luttmer R, De Strooper LM, Berkhof J, et al. Comparing the performance of FAM19A4 methylation analysis, cytology and HPV16/18 genotyping for the detection of cervical (pre)cancer in high-risk HPV-positive women of a gynecologic outpatient population (COMETH study). Int J Cancer 2016; 138:992-1002.

22. Bu Q, Wang S, Ma J, et al. The clinical significance of FAM19A4 methylation in high-risk HPV-positive cervical samples for the detection of cervical (pre)cancer in Chinese women. BMC Cancer 2018; 18:1182.

23. Hernandez JM, Siegel EM, Riggs B, et al. DNA methylation profiling across the spectrum of HPV-associated anal squamous neoplasia. PLoS One 2012; 7:e50533.

24. Zhang J, Martins CR, Fansler ZB, et al. DNA methylation in anal intraepithelial lesions and anal squamous cell carcinoma. Clin Cancer Res 2005; 11:6544-9.

25. Siegel EM, Eschrich S, Winter K, et al. Epigenomic characterization of locally advanced anal cancer: a radiation therapy oncology group 98-11 specimen study. Dis Colon Rectum 2014; 57:941-57.

26. Adams TS, Mbatani NH. Clinical management of women presenting with field effect of HPV and intraepithelial disease. Best Pract Res Clin Obstet Gynaecol 2018; 47:86-94.

27. Bacon MC, von Wyl V, Alden C, et al. The Women's Interagency HIV study: an observational cohort brings clinical sciences to the bench. Clin Diagn Lab Immunol 2005; 12:1013-9.

28. Nayar R, Wilbur DC. The Pap test and Bethesda 2014. Cancer Cytopathology 2015; 123:271-81.

29. Solomon D, Davey D, Kurman R, et al. The 2001 Bethesda system: terminology for reporting results of cervical cytology. JAMA 2002; 287:2114-9.

30. Darragh TM, Colgan TJ, Thomas Cox J, et al; Members of the LAST Project Work Groups. The Lower Anogenital Squamous Terminology Standardization project for HPV-associated lesions: background and consensus recommendations from the College of American Pathologists and the American Society for Colposcopy and Cervical Pathology. Int J Gynecol Pathol 2013; 32:76-115.

31. Hariri S, Unger ER, Sternberg M, et al. Prevalence of genital human papillomavirus among females in the United States, the National Health and Nutrition Examination Survey, 2003-2006. J Infect Dis 2011; 204:566-73.

32. Onyekwuluje JM, Steinau M, Swan DC, Unger ER. A real-time PCR assay for HPV52 detection and viral load quantification. Clin Lab 2012; 58:61-6.

33. Bouvard V, Baan R, Straif K, et al; WHO International Agency for Research on Cancer Monograph Working Group. A review of human carcinogens-part B: biological agents. Lancet Oncol 2009; 10:321-2.

34. Humans IWGotEoCRt. Biological agents. Volume 100 B. A review of human carcinogens. IARC Monogr Eval Carcinog Risks Hum 2012; 100:1-441.

35. Livak KJ, Schmittgen TD. Analysis of relative gene expression data using realtime quantitative PCR and the 2(-Delta Delta C(T)) method. Methods 2001; 25:402-8.

36. Reif SS, Whetten K, Wilson ER, et al. HIV/AIDS in the southern USA: a disproportionate epidemic. AIDS Care 2014; 26:351-9.

37. Oh HY, Kim MK, Seo S, et al. Alcohol consumption and persistent infection of high-risk human papillomavirus. Epidemiol Infect 2015; 143:1442-50.

38. Schabath MB, Thompson ZJ, Egan KM, et al. Alcohol consumption and prevalence of human papillomavirus (HPV) infection among US men in the HPV in Men (HIM) study. Sex Transm Infect 2015; 91:61-7.

39. Sand FL, Munk C, Jensen SM, Svahn MF, Frederiksen K, Kjær SK. Long-term risk for noncervical anogenital cancer in women with previously diagnosed high-grade cervical intraepithelial neoplasia: a Danish Nationwide Cohort Study. Cancer Epidemiol Biomarkers Prev 2016; 25:1090-7.

40. Tong WW, Jin F, McHugh LC, et al. Progression to and spontaneous regression of high-grade anal squamous intraepithelial lesions in HIV-infected and uninfected men. AIDS 2013; 27:2233-43. 